\title{
Donor Notification \& Counseling - Our Experience from a Tertiary Care Hospital in Central India
}

\author{
Authors \\ Dr Anita M. Sardeshpande, Dr Sanjay N. Parate* \\ Department of Blood Bank, Superspeciality Hospital and Govt. Medical College, Nagpur, Maharashtra, \\ India \\ *Corresponding Author \\ Dr Sanjay N. Parate
}

Department of Blood Bank, Superspeciality Hospital \& Govt. Medical College, Nagpur, Maharashtra, India Email: sanjaynparate@gmail.com

\begin{abstract}
Background: Transfusion services in addition to their prime responsibility of supplying safe blood to the patient, also have a responsibility toward donor safety by means of donor notification \& post donation counseling. TTI reactive donor notification is essential for early clinical intervention to minimize their disease and the risk to the partners / close contacts.

Materials \& Methods: The present observational study was carried out in blood bank of Tertiary Care Hospital in Central India over a period of 4 years involving total (8172) donors including 156 reactive donors. The reactive donors were informed by blood bank counselor about an abnormal test result with an advice to report to blood bank for one to one counseling and referral to the respective department for repeat testing and further management. The response rate of transfusion transmitted infection reactive donors after notification of their abnormal test results was evaluated.

Results: During the study period total donation of 8172 units both from voluntary and replacement donors were subjected to routine TTI screening by ELISA. Of these, (156) blood donors were found to be seroreactive. TTI reactive donors (156) for various markers were contacted, 134 (85.9\%) telephonically and the remainder 22 (14.1\%) who could not be contacted on phone were contacted by post maintaining confidentiality. Seroreactivity of 156 contacted donors was (HIV, HCV, HBSAg, syphilis - $17: 47: 91: 1$ ). Of the 134 contacted donors telephonically, response rate was $93.3 \%$ as 125 donors reported. Of the 22 contacted donors by post, 10 donors responded i.e. response rate was 45.4\%. Of the total donors (156), response rate was $86.53 \%$ \& non response rate was $13.4 \%$.

Conclusion: Our study shows that our response rate was increased due to mobile communication. Response rate was also increased due to increased knowledge of infectious diseases which results in self deferral of blood donors belonging to high risk group.

Keywords: Blood donor counseling, donor notification, transfusion - Transmitted infections, HIV, HCV, HBSAg.
\end{abstract}




\section{Introduction}

Although blood transfusion plays an important role in the supportive care of medical and surgical patients, unsafe transfusion practices also put millions of people at risk of transfusion transmissible infection (TTI). ${ }^{(1)}$ The WHO recommends that, atleast, all donated blood should be fully screened for Hepatitis B virus (HBV), Hepatitis C virus (HCV) \& Human immuno deficiency virus (HIV) and syphilis. ${ }^{(2)}$ In India, disclosure of viral Transfusion Transmitted infection reactivity to the blood donor was not permitted until December 2004; at that time, the National Blood Transfusion council, Government of India, formulated strategy for the same. ${ }^{(3)}$ Blood banks are now required to obtain written consent at the time of donation from the donors as to whether they wish to be informed about a reactive test result. They are required to refer donors who tested HIV reactive to the designated voluntary counseling and testing centres for disclosure, counseling and referral. All donors reactive to hepatitis $\mathrm{B}$ or hepatitis $\mathrm{C}$ need to be referred to a gastroenterologist for further management. ${ }^{(4)}$

Transfusion Transmitted infection reactive donor notification is essential for early clinical intervention to minimize their disease and the risk to the partners/close contacts. Reactive donors are intimated telephonically and by post for one-toone counseling and repeat sampling and to elicit any high risk behaviour. This study is carried out to avail information about counseling success rate and referral care \& to assess the attitude of reactive blood donors in response to post donation notification and counseling.

\section{Materials \& Methods}

The present observational study was carried out in blood bank and tertiary care hospital in Central India over a period of 4 years involving total (8172) donors including 156 reactive donors.

\section{Results}

During the year 2014 to 2017 total donation of (8172) units both from voluntary and replacement donors were subjected to routine transfusion Transmitted Infection screening by ELISA. Of these 156 blood donors were found to be seroreactive. Among these 156 seroreactive cases, 17 cases were HIV positive, 91 cases were HBSAg positive, 47 cases were $\mathrm{HCV}$ positive, I case was VDRL positive.

As per age wise distribution, 45 were below 25 years of age, 92 were between 26 and 35 years of age and 19 were above 35 years. The age wise distribution of responded and non-responded donors is given in Figure 1. The gender wise distribution was as follows. There were 153 male and 3 female donors. One Hundred two (65\%) were married and fifty four (35\%) were unmarried. (Figure 2) Geographical distribution of the responded \& non-responded reactive donors is given in Figure 3.

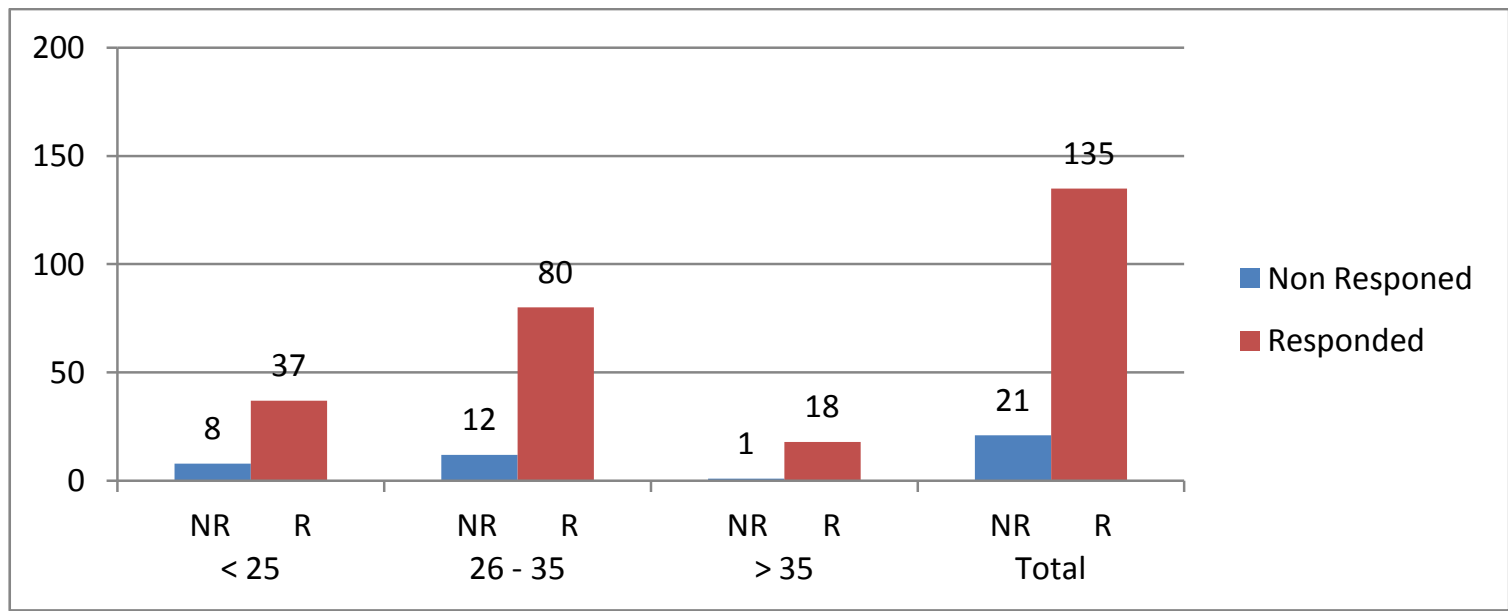

Figure -1 Age distribution of responded and non-responded TTI reactive donors 


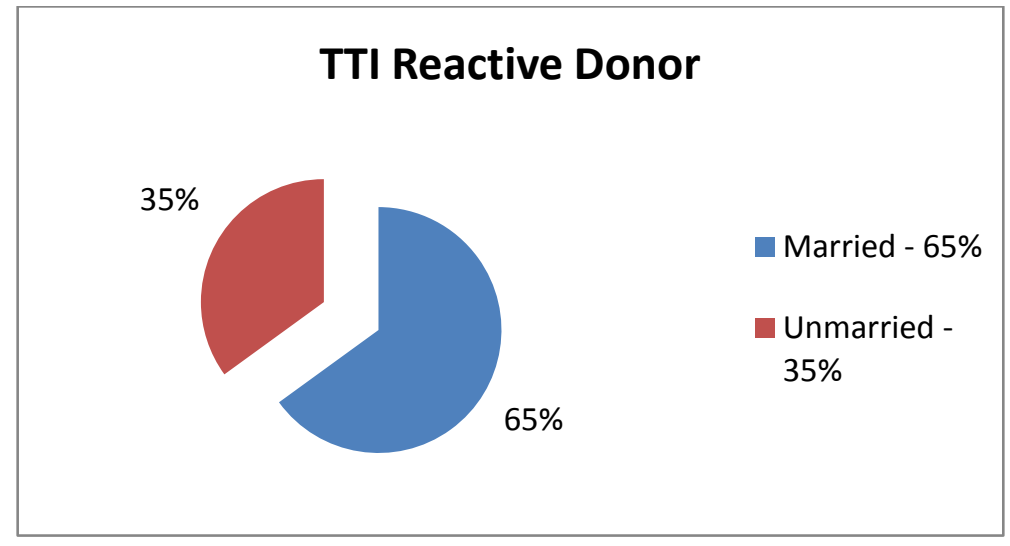

Figure - 2 Pie chart showing the marital status of the TTI reactive donors

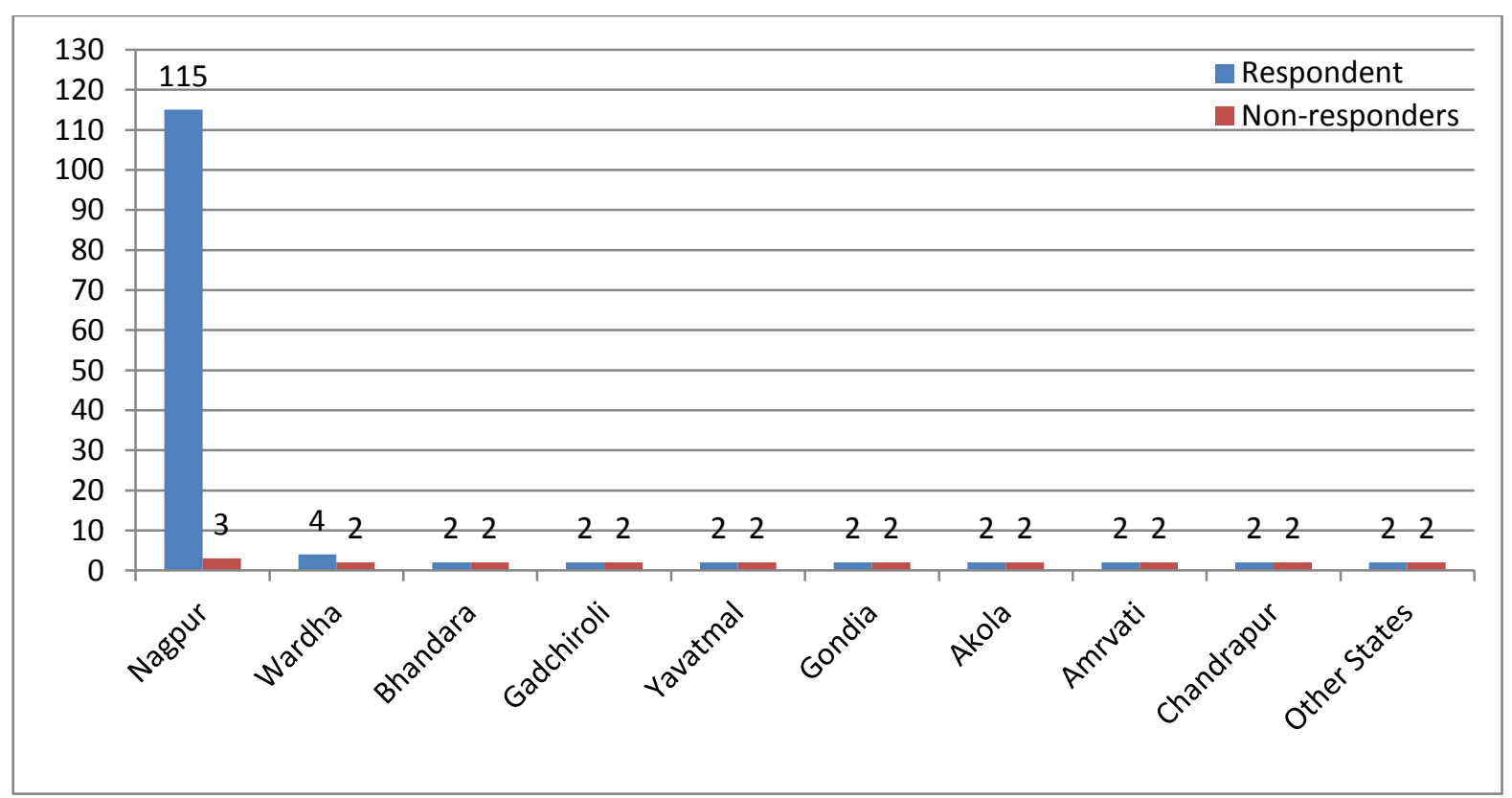

Figure 3 Geographical distribution of responders \& non responders Transfusion Transmitted Infection reactive donors

The TTI reactive donors (156) for various markers were contacted (Figure 4). $134 \quad(85.9 \%)$ telephonically \& remainder $22(14.1 \%)$ who could not be contacted on phone were contacted by post maintaining confidentiality.

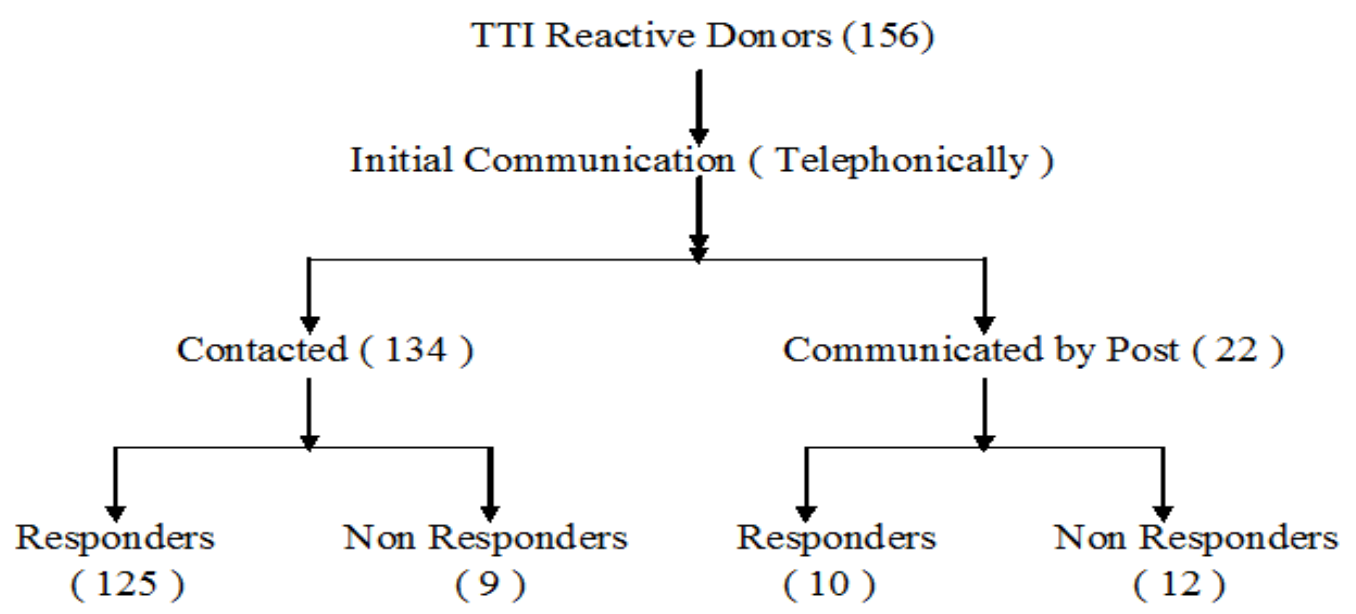

Figure -4 Flow Chart of study results 


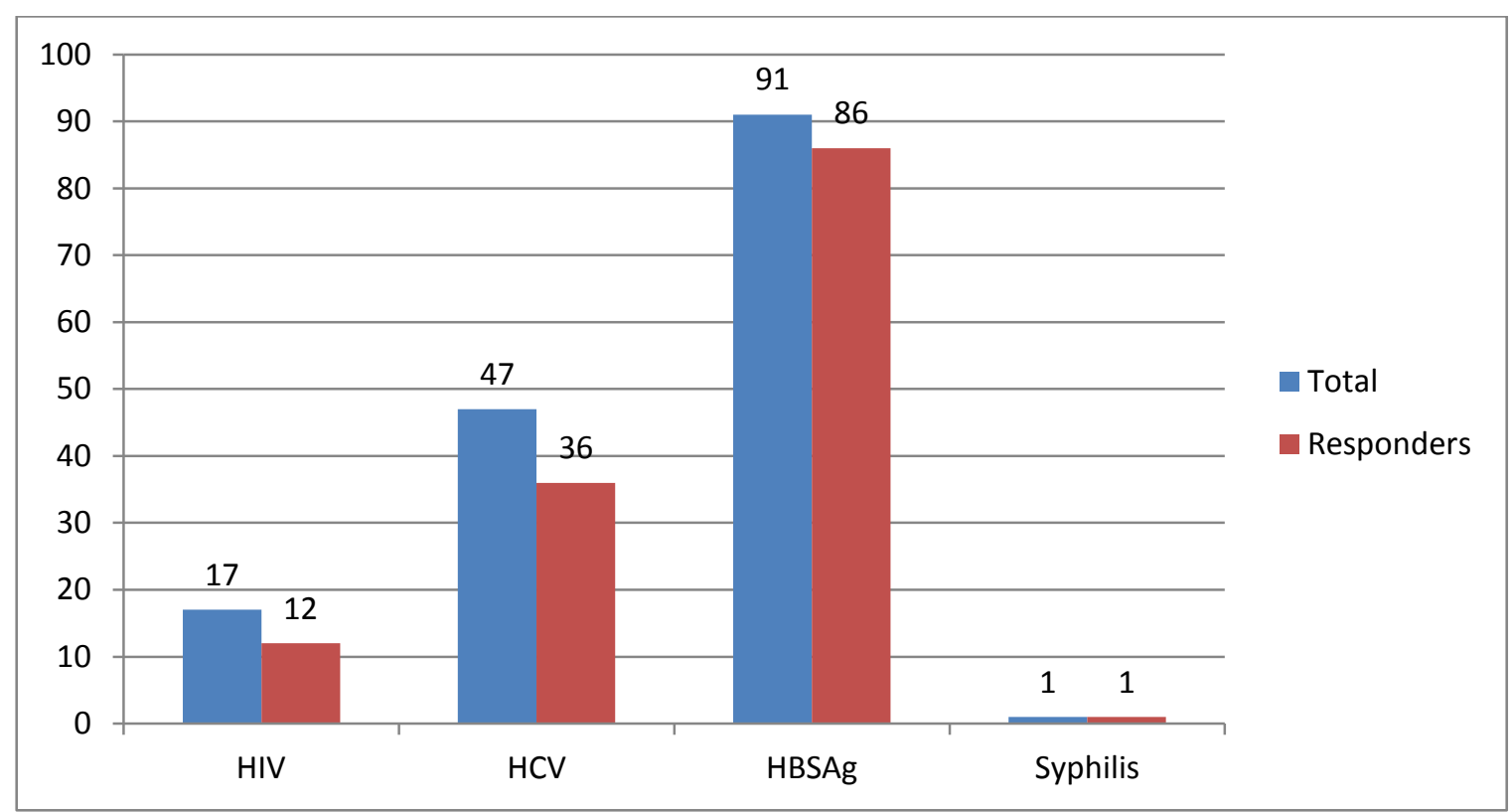

Figure - 5 Response rate according to TTI marker Positivity

Seroreactivity of 135 contacted donors was HIV: HCV : HBSAg : Syphilis - 12:36:86:1. Of the 134 contacted donors by telephone the response rate was $93.3 \%$ as only 125 donors reported (110 on the first, 10 on the second and 5 on the third call ) for one-to-one counseling (Figure 6)

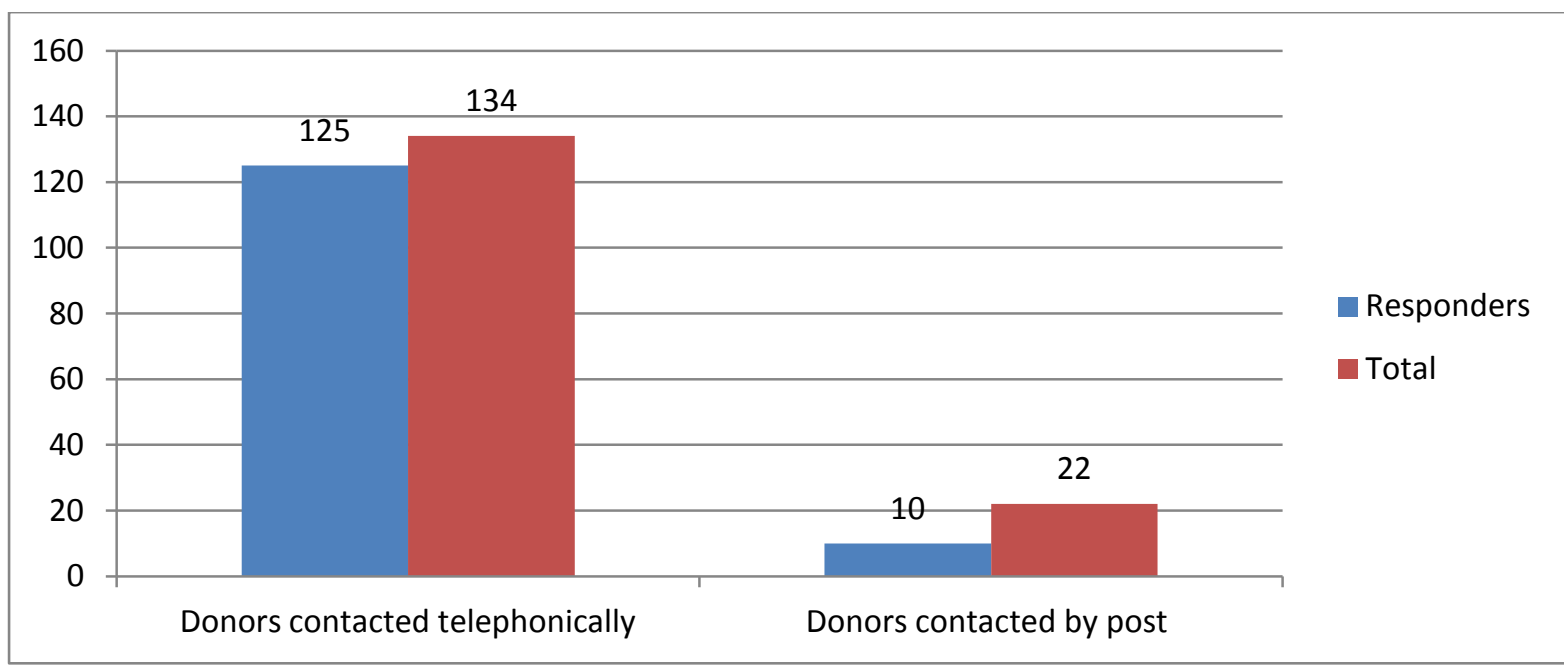

Figure - 6

Among the remaining 21 non responders, 5 were HIV positive, 11 were HCV positive and 5 were HBSAg positive. 10 donors responded by postal communication.
HIV reactive responders were referred to ICTC for counseling and confirmatory testing while $\mathrm{HBV}$ and $\mathrm{HCV}$ reactives were referred to a gastroenterologist for further management.

Table 1 Summary of referral of contacted TTI reactive donors to the concerned specialty for management

\begin{tabular}{|l|c|c|c|c|}
\hline TTI reactive donors & HIV & HCV & HBSAg & Syphilis \\
\hline 1) Total contacted & 17 & 47 & 91 & 1 \\
\hline 2) Responders & 12 & 36 & 86 & 1 \\
\hline 3) Attended the concerned specialty clinic & 12 & 33 & 84 & 1 \\
\hline
\end{tabular}


120 donors among the $135(88.88 \%)$ donors have a positive history of high risk behavior that was not expressed earlier by them during pre-donation counseling and are now on regular treatment for their infection.

The remaining $21(13.4 \% \%)$ donors were nonresponders which is less number. Seroreactivity among these 21 reactive donors was (HIV, HCV, HBSAg, Syphilis $-5: 11: 5: 0$ )

\section{Discussion}

The results of this study show high response rate to blood bank calls to donors with reactive screening tests. These results suggest high health care knowledge and awareness regarding screening tests.

Study carried out at centre (yousuf et al 2007$)^{(5)}$ showed the prevalence of hepatitis B seropositivity was less in regular blood donors compared to first time donors. This implies the need for proper pre-donation counseling of the latter group.

Tynell et al $2007^{(6)}$ also reported a response rate of $88 \%$ in contacted donors as compared to $86.5 \%$ in our study. This high rate reflects the importance of this issue for donors \& their concern for helping others. Other studies have also shown higher response rates of blood donors compared to ours $\left(\right.$ Nilsson Sojka \& Sojka 2003) ${ }^{(7)}$

Lower response rates were also reported (21 to $67 \%$ ) in some studies Moyer et al $1992^{(8)}$, Sanchez et al $2001^{(9)}$, Kleinman et al 2004. ${ }^{(10)}$ In view of the low response rate among relative blood donors, it is important to consider the policy of pre-donation donor screening.

Potential test seekers should be notified of the consequences of providing wrong information at the time of registration. Currently we are relying on good will of blood donors to disclose their information but this has been shown to not be a very effective in one study (Lau et al 2002). ${ }^{(11)}$

Of the 156 contacted donors response rate was $86.5 \%$ as only 135 donors reported from one to one counseling. A large majority of notified donors $(96.3 \%)$ in our study contacted their health care when given clear instructions to do so. These results are encouraging because they indicate that a major element of notification is acted upon when it is worded clearly. Seroreactivity of 156 contacted donors was HIV : HCV : HBSAg : Syphilis $-17: 47: 91: 1$. Among the remaining 21 non responders, 5 was HIV, 11 were $\mathrm{HCV}, 5$ were HSBAg. The very high response rate of contacted donors ensured their concern for knowing their test result status.

\section{Conclusion}

In summary response rate was increased due to mobile communication and increased knowledge regarding the transmission of infectious diseases. This results in self deferral in blood donors belonging to a high risk group. Loss to follow up of reactive blood donors can be minimized by proper pre-donation counseling. Public health authorities should make it mandatory, that every blood donor with a reactive test should contact a health worker of blood bank for further investigation. One counselor in blood bank should be appointed to each relative case, this will result in better compliance and protect the confidentiality of donors.

We recommend further studies regarding the donors understanding of the screening process and factors contributing to donors responses to calls from blood bank.

\section{Reference}

1. Diro E, Alemu S, G / Yohannes A. Blood safety and prevalence of transfusion transmissible viral infections among donors at the Red Cross Blood Bank in Gondar University Hospital. EthoP Med. J. 2008; 46 : 7-13 (PubMed)

2. Blood safety and Donation 2008 June. Fact sheet No. 279 ( Last accessed on 2008 Sep. 2) Available from: http://www.who.int/mediacentre/factsheet/ ps279

3. National Aids Control Organisation. National Blood Policy of India (Last 
accessed on 2012 Aug 27) Available from http://www.unpanl.unorg/intradoc/groups/ public/documents/ pdf.

4. Dontula S, Mathur A, Kamaladoss $\mathrm{T}$, Adimurthy S, Jagannathan L, Donor disclosure - a donor's right and blood bank's responsibility. Transf alter Transf Med. 2012; 12 : 44-50.

5. Yousuf R, Rapiaah M, Ahmed S A et al, Trends in hepatitis B, virus infection among blood donors in Kelantan Malaysia: A retrospective study, south east Asian J. Trop Med Public Health 2007; 38: 1070-4.

6. Tynell E, Norda R, Ekermo B, Sanner M, Andersson S, Bjorkman A, False reactive microbiologic screening test results in Swedish blood donors - How big is the problem ? A survey among blood centres and deferred donors. Transfusion 2007; 47 : 80-9.

7. Nilsson, Sojka B. Sojka P. The blood donation experience perceived, physical, psychological and social impact of blood donation on the donor. Vox Sang 2003; 84 : 120-8.

8. Moyar LA, Shapiro CN, Shulman G, Brugliera PD, Alter MJ. A survey of Hepatitis B surface antigen positive blood donors: Degree of understanding and action taken after notification. Transfusion 1992; 32 : 702-6.

9. Sanchez AM, Ameti DI, Schreiber GB et al. The potential impact of incentives on future blood donation behavior. Transfusion 2001; 41 : 172-8.

10. Kleinman S, Wang B, Wu Y et al. The donor notification process from the donor's perspective. Transfusion 2004; 44: 658-66.

11. Lau JT, Thomas J, Lin CK, HIV related behaviors among voluntary blood donors in Hong Kong. AIDS Care 2002; 14 : 48192. 